\title{
Symmetry and Shape Coexistence in ${ }^{10} \mathrm{Be}$
}

\author{
M.A. Caprio ${ }^{1}$, A.E. McCoy ${ }^{2}$, P.J. Fasano ${ }^{1}$, T. Dytrych ${ }^{3,4}$ \\ ${ }^{1}$ Department of Physics, University of Notre Dame, Notre Dame, \\ Indiana 46556-5670, USA \\ ${ }^{2}$ Institute for Nuclear Theory, University of Washington, Seattle, \\ Washington 98195-1550, USA \\ ${ }^{3}$ Nuclear Physics Institute of the Czech Academy of Sciences, \\ 25068 Řež, Czech Republic \\ ${ }^{4}$ Department of Physics and Astronomy, Baton Rouge, \\ Louisiana State University, Louisiana 70803, USA \\ doi: https://doi.org/10.55318/bgjp.2022.49.1.057
}

\begin{abstract}
Within the low-lying spectrum of ${ }^{10} \mathrm{Be}$, multiple rotational bands are found, with strikingly different moments of inertia. A proposed interpretation has been that these bands variously represent triaxial rotation and prolate axially-deformed rotation. The bands are wellreproduced in $a b$ initio no-core configuration interaction (NCCI) calculations. We use the calculated wave functions to elucidate the nuclear shapes underlying these bands, by examining the Elliott $\mathrm{SU}(3)$ symmetry content of these wave functions. The $a b$ initio results support an interpretation in which the ground-state band, along with an accompanying $K=2$ side band, represent a triaxial rotor, arising from an $\mathrm{SU}(3)$ irreducible representation in the $0 \hbar \omega$ space. Then, the lowest excited $K=0$ band represents a prolate rotor, arising from an $\mathrm{SU}(3)$ irreducible representation in the $2 \hbar \omega$ space.
\end{abstract}

KEY WORDS: Ab initio nuclear theory, no-core configuration interaction (NCCI), no-core shell model (NCSM), shape coexistence, nuclear rotation, Elliott $\mathrm{SU}(3)$ symmetry, Daejeon16 interaction

\section{Introduction}

The low-lying positive-parity spectrum of ${ }^{10} \mathrm{Be}$ contains rotational bands of decidedly different moments of inertia $[1,2]$. A ground state $K=0$ band and excited $K=0$ band (at $\approx 6.18 \mathrm{MeV}$ ) are both observed, through their $4^{+}$members [2-4], and the moment of inertia for the excited band is higher by a factor of $\approx 3$. Indeed, the difference of momenta of inertia is such that the excited band hits the yrast line at $J=4$, and the $4_{1}^{+}$state is a member of the excited band.

Moreover, the ground-state band is accompanied by a proposed $K=2$ side band (at $\approx 5.96 \mathrm{MeV}$ ), observed through its $3^{+}$member [2]. This band has a moment of inertia essentially identical to that of the ground-state band. Comparing the 


\section{M.A. Caprio, A.E. McCoy, P.J. Fasano, T. Dytrych}

excitation energy of the $2^{+}$band head with that of the $2^{+}$member of the groundstate band gives $E\left(2_{2}^{+}\right) / E\left(2_{1}^{+}\right) \approx 1.8$. Such a low ratio is suggestive of a triaxial rotor with maximal triaxiality $\left(\gamma \approx 30^{\circ}\right)[5,6]$.

The cluster interpretation provided by antisymmetrized molecular dynamics (AMD) calculations [7-9] is that ${ }^{10} \mathrm{Be}$ consists of an $\alpha+\alpha$ dimer $\left({ }^{8} \mathrm{Be}\right)$ plus two valence neutrons occupying molecular orbitals. In the ground-state and side bands, these two neutrons occupy equatorial orbitals around the molecular axis ( $\pi$ orbitals), and proton-neutron triaxiality arises, in which an oblate distribution for the neutrons combines with the prolate distribution of the protons (in the $\alpha+\alpha$ dimer) to form an overall triaxial shape [7,9]. In the excited $K=0$ band, the two valence neutrons instead occupy polar orbitals along the molecular axis ( $\sigma$ orbitals), which give rise to a large axially-symmetric prolate deformation.

We turn here to $a b$ initio no-core configuration interaction (NCCI) [10,11], or no-core shell model (NCSM), calculations for ${ }^{10} \mathrm{Be}$ for further insight into the nature of these states. Ab initio results can provide access to traditional collective observables, such as electromagnetic transition strengths, which might otherwise not be readily accessible to experiment [12-14]. Moreover, ab initio calculated wave functions have been found $[15,16]$ to have dominant components with specific Elliott $\mathrm{SU}(3)[17,18]$ or $\mathrm{Sp}(3, \mathbb{R})$ [19] symmetry. Ab initio calculations can thus give insight into the nature of collective states by exposing the symmetry structure of calculated wave functions [20-22].

In a contribution [23] to the proceedings of the previous workshop in this series, we explored the rotational features of the $a b$ initio calculated spectrum of ${ }^{10} \mathrm{Be}$. The $a b$ initio NCCI calculated spectrum from Ref. [23], obtained with the Daejeon16 interaction [24], may be seen in Figure 1 (left). The ground-state band $\left(0^{+}, 2^{+}, 4^{+}\right)$and side band $\left(2^{+}, 3^{+}\right)$are robustly reproduced, terminating at the same angular momentum as in experiment. An excited $K=0$ band with higher moment of inertia is also obtained, likewise in agreement with experiment, but extending well past the last experimentally observed band member, terminating rather at $8^{+}$. A very similar band structure is obtained with other choices of internucleon interaction [13].

Since these calculations are carried out in an oscillator basis, it was straightforward to examine the contributions of configurations with different numbers $N_{\text {ex }}$ of oscillator excitations, shown in Figure 1 (right). For the ground-state and side band members, such as the $0_{1}^{+}$state [Figure 1 (right,a)], the dominant contribution comes from $N_{\mathrm{ex}}=0$ (or " $0 \hbar \omega$ ") configurations, while, for members of the excited $K=0$ band, such as the $0_{2}^{+}$state [Figure 1 (right,b)], the dominant contribution comes from $N_{\mathrm{ex}}=2$ (or " $2 \hbar \omega$ ") configurations.

Here, we furthermore extract the Elliott $\mathrm{SU}(3)$ symmetry structure of the same calculated wave functions considered in Ref. [23], to see what this can elucidate regarding the nature of the bands and their intrinsic nuclear shapes. What does the $\mathrm{SU}(3)$ structure tell us about the deformation? Do the ground-state band and 
Symmetry and Shape Coexistence in ${ }^{10} \mathrm{Be}$
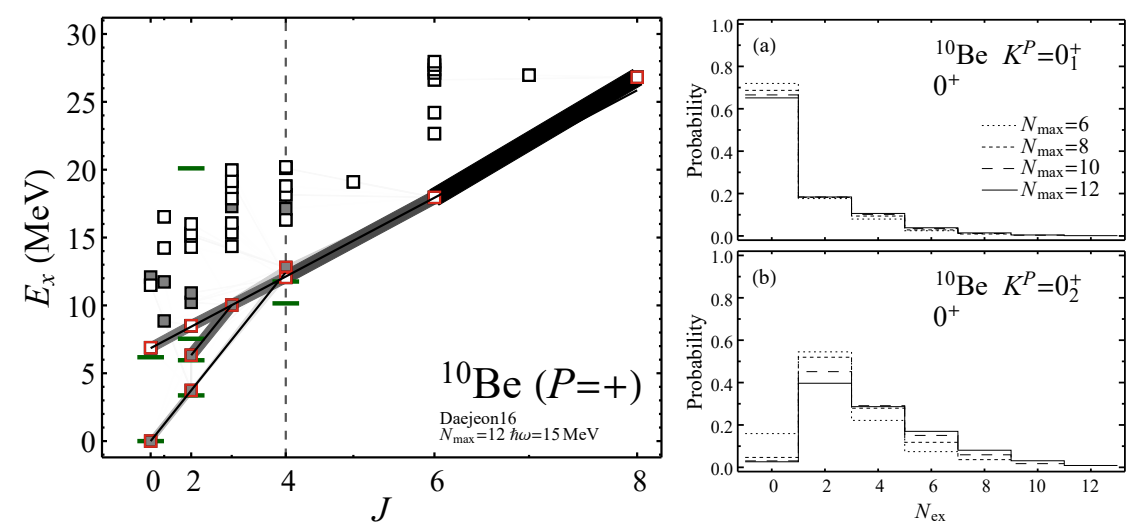

Figure 1. (Left) Ab initio calculated energy spectrum of positive parity states for ${ }^{10} \mathrm{Be}$, for $N_{\max }=12$. The $E 2$ transition strengths (indicated by line widths) are shown for transitions from members of the ground-state band $(K=0)$, side band $(K=2)$, and excited $K=0$ band. States are classified as $0 \hbar \omega$ (filled squares) or $2 \hbar \omega$ (open squares) by their $N_{\text {ex }}$ decomposition. Experimental energies (thick horizontal lines), rotational energy fits (thin lines), and the maximal angular momentum for the $0 \hbar \omega$ space (vertical dashed line) are shown for comparison. (Right) Decompositions with respect to $N_{\text {ex }}$, for the calculated $0^{+}$band head states of the (a) ground-state band and (b) long band. Shown for $N_{\max }=6$ to 12 (dotted through solid curves). Figure adapted from Ref. [23].

side band together represent the spectrum of a triaxial rotor? After introducing the $\mathrm{SU}(3)$ irreducible representations (irreps) arising in the shell-model space for ${ }^{10} \mathrm{Be}$ (Section 2), we present results for the symmetry decompositions of the rotational states in ${ }^{10} \mathrm{Be}$ and discuss their implications (Section 3).

\section{$2 \mathrm{SU}(3)$ Content of the Many-Body Space for ${ }^{10} \mathrm{Be}$}

The nuclear many-body space reduces into irreducible representations (irreps) of definite $\mathrm{U}(3) \times \mathrm{SU}(2)$ symmetry, described by the simultaneous quantum numbers $N_{\mathrm{ex}}(\lambda, \mu) S$. Here $\mathrm{U}(3)$ is the symmetry group of the harmonic oscillator. Its irreps may be labeled by $\mathrm{U}(1) \times \mathrm{SU}(3)$ quantum numbers, where the $\mathrm{U}(1)$ group is generated by the harmonic oscillator Hamiltonian, and yields the number of oscillator quanta as its quantum number (we take $N_{\mathrm{ex}}$ relative to the lowest Pauli-allowed oscillator configuration), while the $\mathrm{SU}(3)$ group, generated by the orbital angular momentum operators $L_{1, M}$ and Elliott's quadruople operators $\mathcal{Q}_{2, M}$ (see, e.g., Appendix A of Ref. [25]), gives the Elliott $(\lambda, \mu)$ quantum numbers. These operators mutually commute with the spin angular momentum operators $S_{1, M}$, which generate the usual spin $\mathrm{SU}(2)$ group.

The $\mathrm{U}(3) \times \mathrm{SU}(2)$ irreps arising in the many-body space for a nucleus may be deduced by general methods [26] which are implemented in the code 


\section{M.A. Caprio, A.E. McCoy, P.J. Fasano, T. Dytrych}

LSU3shell [27]. For ${ }^{10} \mathrm{Be}$, irreps from the $0 \hbar \omega$ space (and selected irreps from the $2 \hbar \omega$ space) are listed in Table 1 .

Table 1 . The $\mathrm{U}(3) \times \mathrm{SU}(2)$ irreps arising in the $0 \hbar \omega$ space for ${ }^{10} \mathrm{Be}$, ordered by increasing eigenvalue of the $\mathrm{SU}(3)$ Casimir operator, together with selected irreps in the $2 \hbar \omega$ space.

\begin{tabular}{lcc}
\hline \hline$N_{\mathrm{ex}}(\lambda, \mu)$ & $S$ & $\left\langle C_{\mathrm{SU}(3)}\right\rangle$ \\
\hline $0(0,0)$ & $0,1,2$ & 0 \\
$0(1,1)$ & $0,1,2$ & 6 \\
$0(0,3)$ & 1 & 12 \\
$0(3,0)$ & 1 & 12 \\
$0(2,2)$ & 0 & 16 \\
$\cdots$ & & \\
$2(6,1)$ & $0,1,2$ & 42.67 \\
$2(8,0)$ & 0 & 58.67 \\
\hline \hline
\end{tabular}

The irrep within the many-body space having the largest eigenvalue $\left\langle C_{\mathrm{SU}(3)}\right\rangle$, of the $\mathrm{SU}(3)$ quadratic Casimir operator $C_{\mathrm{SU}(3)}=\frac{1}{6}(\mathcal{Q} \cdot \mathcal{Q}+3 \mathbf{L} \cdot \mathbf{L})$, is known as the leading irrep. This is the irrep which is most strongly bound (energetically preferred) by Elliott's schematic $-\mathcal{Q} \cdot \mathcal{Q}$ Hamiltonian [18]. The concept of a leading irrep was defined in the context of the traditional shell model, where consideration was limited to the irreps in the $0 \hbar \omega$ space. However, we may similarly identify a leading irrep in the $2 \hbar \omega$ space, etc. For ${ }^{10} \mathrm{Be}$, the eigenvalues $\left\langle C_{\mathrm{SU}(3)}\right\rangle=\frac{2}{3}\left(\lambda^{2}+\lambda \mu+\mu^{2}+3 \lambda+3 \mu\right)$ are shown in Table 1 as well.

To each irrep of $\mathrm{SU}(3)$ is also associated a deformation $(\beta, \gamma)$, given by [28]

$$
\beta^{2}=\frac{4 \pi}{5\left(A \overline{r^{2}}\right)^{2}}\left(\lambda^{2}+\lambda \mu+\mu^{2}+3 \lambda+3 \mu+3\right) \quad \tan \gamma=\frac{\sqrt{3}(\mu+1)}{2 \lambda+\mu+3} .
$$

From this expression (1), we can see that the leading irrep furthermore has the largest $\beta$ deformation. The deformations $(\beta, \gamma)$ for the $0 \hbar \omega$ and $2 \hbar \omega$ irreps of ${ }^{10} \mathrm{Be}$ are shown on a polar plot in Figure 2.
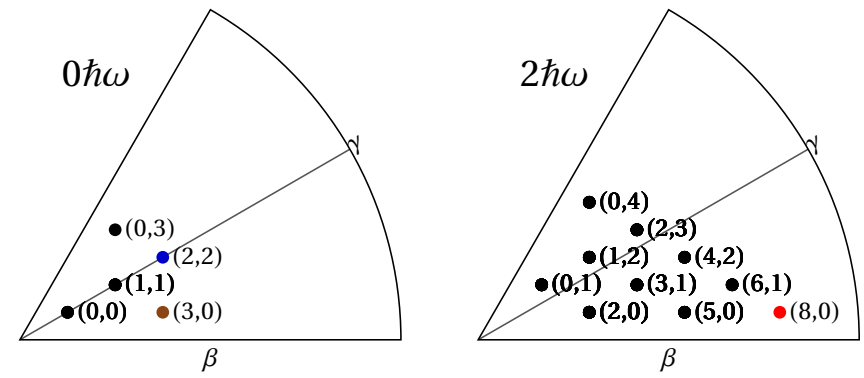

Figure 2. Deformation $(\beta, \gamma)$ parameters for the $\mathrm{U}(3)$ irreps in the $0 \hbar \omega$ (left) and $2 \hbar \omega$ (right) spaces for ${ }^{10} \mathrm{Be}$. 
For ${ }^{10} \mathrm{Be}$, the leading irrep in the $0 \hbar \omega$ space (see Table 1 and Figure 2 ) has quantum numbers $N_{\text {ex }}(\lambda, \mu) S=0(2,2) 0$, while the two subleading irreps, degenerate with respect to the Casimir operator, have quantum numbers $0(3,0) 1$ and $0(0,3) 1$. Irreps with quantum numbers $(\lambda, 0)$ or $(0, \mu)$, such as $(3,0)$ and $(0,3)$, have (nearly) prolate $\left(\gamma \approx 0^{\circ}\right)$ and (nearly) oblate $\left(\gamma \approx 60^{\circ}\right)$ deformations, respectively, while those with quantum numbers $(\lambda, \mu=\lambda)$, such as $(2,2)$, have a maximally triaxial deformation $\left(\gamma=30^{\circ}\right)$. Then, the leading irrep within the $2 \hbar \omega$ space for ${ }^{10} \mathrm{Be}$ has $N_{\mathrm{ex}}(\lambda, \mu) S=2(8,0) 0$. This irrep again is identified with a (nearly) prolate deformation, larger than for the $0 \hbar \omega$ irreps.

The levels within these irreps are obtained by considering the standard $\mathrm{SU}(3) \rightarrow$ $\mathrm{SO}(3)$ branching relations, giving the allowed values for the orbital angular momentum $L$ [18], which then couples with the spin to give the total angular momentum $J(L \times S \rightarrow J)$, as shown in Figure 3. The angular momentum content of the leading $0(2,2) 0$ irrep exactly matches that of the combined ground-state and side bands in ${ }^{10} \mathrm{Be}$ (the assignment of the $L=4$ terminating state to one band or the other, in the branching rule, is arbitrary). Then, an $(8,0) \mathrm{SU}(3)$ irrep contains a single $K_{L}=0$ band, extending through $L=8$. The angular momentum content thus, notably, matches that of the $a b$ initio calculated $2 \hbar \omega$ excited $K=0$ band.

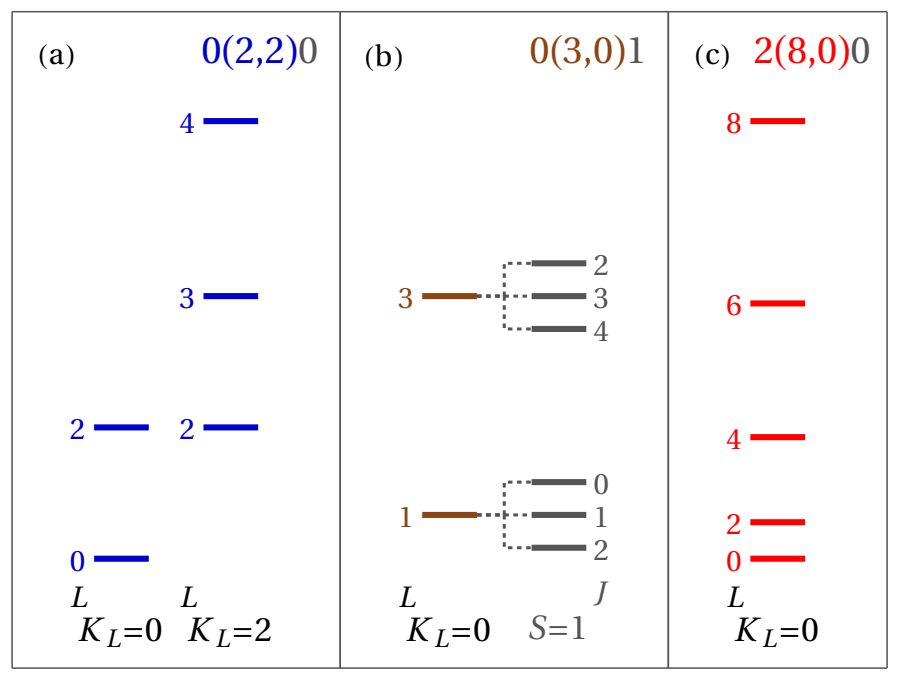

Figure 3. Energy levels within the $\mathrm{U}(3)$ irreps relevant to the low-lying positive-parity spectrum of ${ }^{10} \mathrm{Be}$ : (a) the leading irrep in the $0 \hbar \omega$ space $\left[N_{\mathrm{ex}}(\lambda, \mu) S=0(2,2) 0\right]$, (b) the subleading irrep in the $0 \hbar \omega$ space [identical spectra are obtained for $0(3,0) 1$ and $0(0,3) 1]$, and (c) the leading irrep of the $2 \hbar \omega$ space $[2(8,0) 0]$. In panel (b), the orbital angular momenta $L$ arising within the $\mathrm{U}(3)$ irrep are shown at left, and the resultant total angular momenta $J$ from coupling with spin $S=1$ are shown at right. 


\section{M.A. Caprio, A.E. McCoy, P.J. Fasano, T. Dytrych}

\section{$3 \mathrm{SU}(3)$ Nature of the Low-Lying States of ${ }^{10} \mathrm{Be}$}

To decompose the ab initio calculated wave function into contributions from different $\mathrm{U}(3) \times \mathrm{SU}(2)$ symmetry subspaces, with quantum numbers $N_{\mathrm{ex}}(\lambda, \mu) S$, we make use of the "Lanczos trick" [29]. In general, this technique provides the decomposition of a wave function into its projections onto the eigenspaces of a given Hermitian operator. Thus, to obtain a decomposition with respect to $L$ or $S$, one decomposes the wave function into its projections onto eigenspaces of the $\mathbf{L}^{2}$ or $\mathbf{S}^{2}$ operators [30], respectively. For a decomposition with respect to $\mathrm{SU}(3)$ quantum numbers $(\lambda, \mu)$, one decomposes the wave function into its projections onto eigenspaces of the $\mathrm{SU}(3)$ quadruatic Casimir operator $[22,31,32]$. (This may still leave some unresolved degeneracies if different irreps share the same eigenvalue for $C_{\mathrm{SU}(3)}$.)

Here, to obtain simultaneous decompositions with respect to the full set of quantum numbers $N_{\mathrm{ex}}(\lambda, \mu) S$, we decompose the wave functions into the eigenspaces of an operator constructed as a linear combination

$$
C=a_{\mathrm{U}(1)} N_{\mathrm{ex}}+a_{\mathrm{SU}(3)} C_{\mathrm{SU}(3)}+a_{\mathrm{SU}(2)} \mathbf{S}^{2} .
$$

The coefficients $a_{i}$ are chosen so as to avoid numerical near-degeneracies in eigenvalues for this operator within the ${ }^{10} \mathrm{Be}$ NCCI model space. ${ }^{1}$ Both the initial diagonalization and the subsequent Lanczos decomposition have been carried out with the code MFDn [34,35].

The resulting probability decompositions are shown for members of the various bands in Figure 4. These decompositions are for wave functions calculated with $N_{\max }=8$. For this lower $N_{\max }$, note that the calculated excited $K=0$ band still lies entirely above the ground-state band. Thus the ground-state band member is $4_{1}^{+}$, and the excited $K=0$ band member is $4_{2}^{+}$, opposite to the situation in Figure 1 (and experiment). In all these states, the dominant contributions at low $N_{\text {ex }}$ are dressed by contributions at higher $N_{\text {ex }}$, which account for about half of the norm [recall Figure 1 (right)].

The ground-state band [Figure 4 (top)] and side band [Figure 4 (middle)] members all have as their single largest component the leading $0(2,2) 0$ (triaxial) irrep of the $0 \hbar \omega$ space. The side band members are comparatively "pure", with nearly the entire $N_{\text {ex }}=0$ contribution coming from $0(2,2) 0$, while the ground-state band members have significant admixtures of the subleading $0(3,0) 1$ (prolate) and/or $0(0,3) 1$ (oblate) irreps. Despite these secondary contributions, the overall predominance of $0(2,2) 0$ across these states provides a microscopic justification for the interpretation of these bands in terms of a triaxial rotor spectrum.

\footnotetext{
${ }^{1}$ In fact, the decomposition which was carried out also included the proton spin $S_{p}$ and neutron spin $S_{n}$ among the set of simultaneous quantum numbers (see, e.g., Ref. [33]), through inclusion of terms proportional to $\mathbf{S}_{p}^{2}$ and $\mathbf{S}_{n}^{2}$ in the operator (2) with respect to which the decomposition is carried out. Resolving $S_{p}$ and $S_{n}$ helps to lift certain degeneracies, e.g., although the $0(3,0) 1$ and $0(0,3) 1$ irreps for ${ }^{10}$ Be share the same eigenvalue of $C_{\mathrm{SU}(3)}$, they differ in whether their spin arises from the neutrons or protons, respectively.
} 
Symmetry and Shape Coexistence in ${ }^{10} \mathrm{Be}$
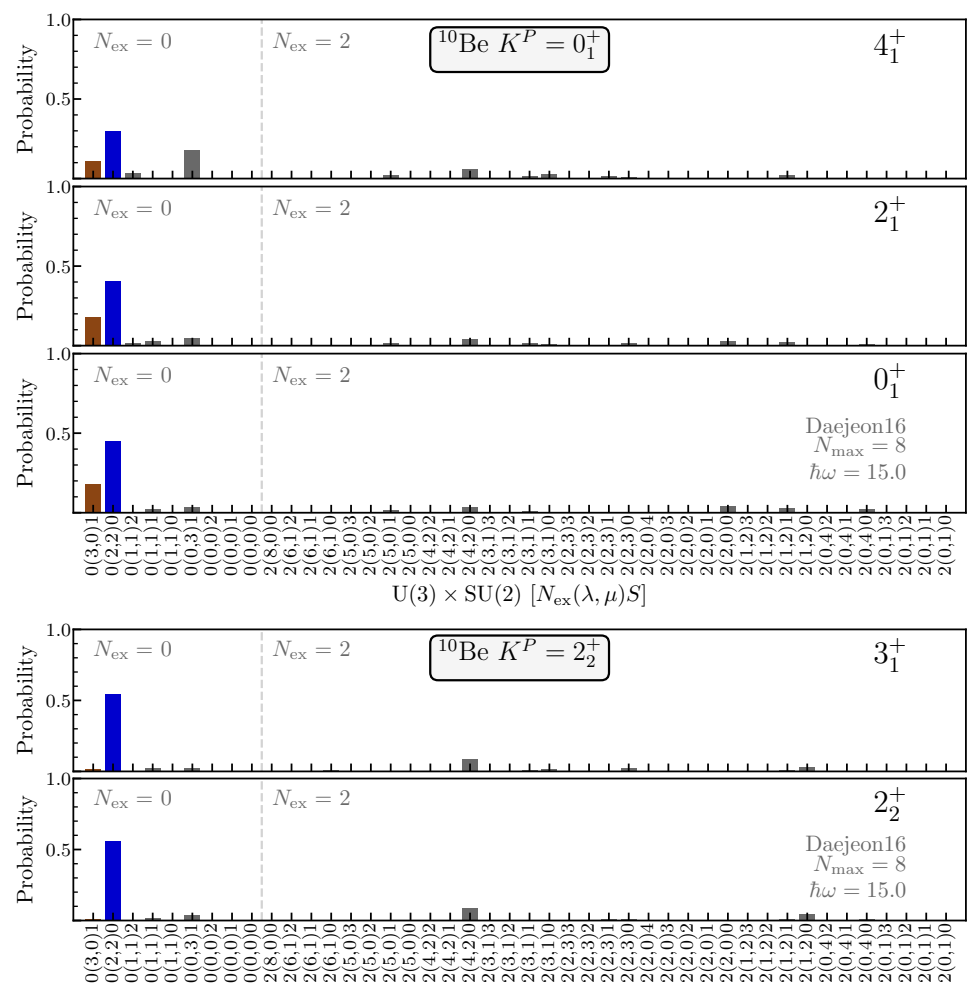

$\mathrm{U}(3) \times \mathrm{SU}(2)\left[N_{\mathrm{ex}}(\lambda, \mu) S\right]$

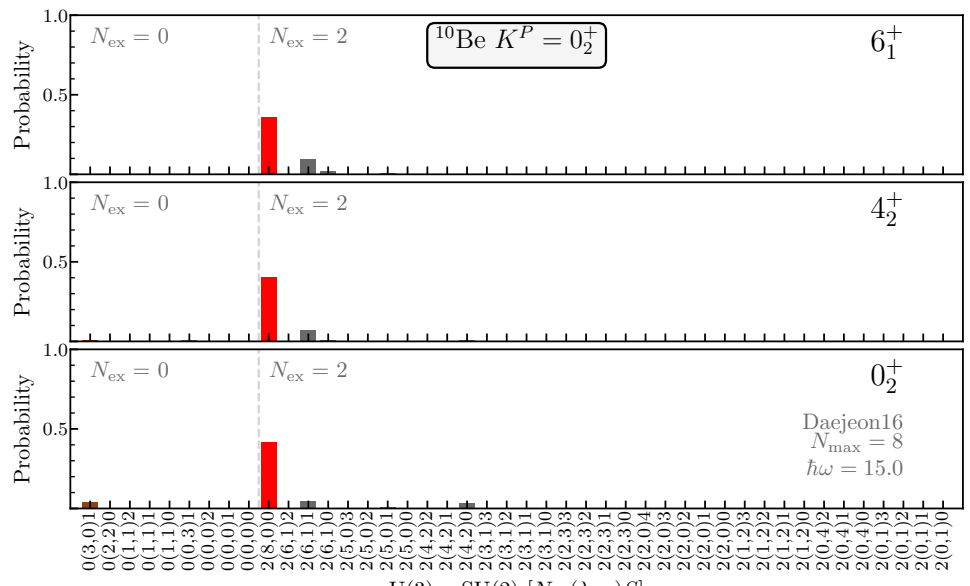

$\mathrm{U}(3) \times \mathrm{SU}(2)\left[N_{\mathrm{ex}}(\lambda, \mu) S\right.$

Figure 4. Decompositions with respect to $\mathrm{U}(3)$ and spin quantum numbers for members of the ground-state band (top), side band (middle), and excited $K=0$ band (bottom) of ${ }^{10} \mathrm{Be}$, calculated with $N_{\max }=8$. 


\section{M.A. Caprio, A.E. McCoy, P.J. Fasano, T. Dytrych}

Moreover, in ${ }^{10} \mathrm{Be}$, the $0(2,2) 0$ irrep is obtained by coupling a proton $(2,0)$ (prolate) irrep with a neutron $(0,2)$ (oblate) irrep. This underlying structure for the $(2,2)$ irrep supports the interpretation of triaxiality in ${ }^{10} \mathrm{Be}$ as being protonneutron in nature, arising from the combination of prolate proton and oblate neutron distributions.

The decompositions for the excited $K=0$ band members [Figure 4 (bottom)], in turn, all have the leading $2(8,0) 0$ (prolate) irrep of the $2 \hbar \omega$ space as by far their strongest single contribution. This irrep is obtained in the ${ }^{10} \mathrm{Be}$ many-body space by coupling a weakly prolate proton $(2,0)$ irrep with a more strongly prolate neutron $(6,0)$ irrep, supporting the interpretation that the increased deformation of the excited $K=0$ band is contributed primarily by the neutrons.

\section{Conclusion}

The rotational bands in the low-lying positive-parity spectrum of ${ }^{10} \mathrm{Be}-$ as obtained from experiment, microscopic AMD calculations, and ab initio NCCI calculations - provide an example of shape coexistence. A modestly deformed triaxial rotor coexists with a more strongly deformed prolate rotor. In a shellmodel interpretation, the states composing the former structure are $0 \hbar \omega$ states, while those of the latter structure are $2 \hbar \omega$ states, both dressed by higher oscillator excitations.

Decompositions of the NCCI wave functions, into contributions defined by U(3) and spin quantum numbers, corroborate and elaborate upon this picture. The leading $\mathrm{SU}(3)$ irreps of the $0 \hbar \omega$ and $2 \hbar \omega$ spaces $-(2,2)$ and $(8,0)$, respectively - are found to define the band structure of the low-lying spectrum and to provide the strongest contributions to the calculated states. The identification of the ground-state and side bands with the $(2,2)$ irrep, in particular, supports their interpetation as representing triaxial rotation.

\section{Acknowledgements}

Pieter Maris and James P. Vary are gratefully acknowledged for valuable discussions and for their collaboration on the calculations of Ref. [23]. This material is based upon work supported by the U.S. Department of Energy, Office of Science, under Award Numbers DE-FG02-95ER40934 and DE-FG02-00ER41132. This research used computational resources of the National Energy Research Scientific Computing Center (NERSC), a U.S. Department of Energy, Office of Science, user facility supported under Contract DE-AC02-05CH11231. 
Symmetry and Shape Coexistence in ${ }^{10} \mathrm{Be}$

\section{References}

[1] M. Freer (2007) Rep. Prog. Phys. 702149.

[2] H.G. Bohlen, T. Dorsch, Tz. Kokalova, W. von Oertzen, Ch. Schulz, C. Wheldon (2007) Phys. Rev. C 75054604.

[3] M. Freer, E. Casarejos, L. Achouri, C. Angulo, N.I. Ashwood, N. Curtis, P. Demaret, C. Harlin, B. Laurent, M. Milin, N.A. Orr, D. Price, R. Raabe, N. Soić, V.A. Ziman (2006) Phys. Rev. Lett. 96042501.

[4] D. Suzuki, A. Shore, W. Mittig, J.J. Kolata, D. Bazin, M. Ford, T. Ahn, D. Becchetti, S. Beceiro Novo, D. Ben Ali, B. Bucher, J. Browne, X. Fang, M. Febbraro, A. Fritsch, E. Galyaev, A.M. Howard, N. Keeley, W.G. Lynch, M. Ojaruega, A.L. Roberts, X.D. Tang (2013) Phys. Rev. C 87054301.

[5] A.S. Davydov, G.F. Filippov (1958) Nucl. Phys. 8237.

[6] J. Meyer-ter-Vehn (1975) Nucl. Phys. A 249111.

[7] Y. Kanada-En'yo, H. Horiuchi (1997) Phys. Rev. C 552860.

[8] Y. Kanada-En'yo, H. Horiuchi, A. Doté (1999) Phys. Rev. C 60064304.

[9] T. Suhara, Y. Kanada-En'yo (2010) Prog. Theor. Phys. 123303.

[10] P. Navrátil, J.P.Vary, B.R. Barrett (2000) Phys. Rev. Lett. 845728.

[11] B.R. Barrett, P. Navrátil, J.P. Vary (2013) Prog. Part. Nucl. Phys. 69131.

[12] M.A. Caprio, P. Maris, J.P. Vary (2013) Phys. Lett. B 719179.

[13] P. Maris, M.A. Caprio, J.P. Vary (2015) Phys. Rev. C 91014310.

[14] M.A. Caprio, P. Maris, J.P. Vary, R. Smith (22015) Int. J. Mod. Phys. E 241541002.

[15] T. Dytrych, K.D. Sviratcheva, C. Bahri, J.P. Draayer, J.P. Vary (2007) Phys. Rev. Lett. 98162503.

[16] T. Dytrych, K.D. Launey, J.P. Draayer, P. Maris, J.P. Vary, E. Saule, U. Catalyurek, M. Sosonkina, D. Langr, M.A. Caprio (2013) Phys. Rev. Lett. 111252501.

[17] J.P. Elliott (1958) Proc. R. Soc. London A 245128.

[18] M. Harvey (1968) The nuclear $S U_{3}$ model. In: "Advances in Nuclear Physics", Vol. 1, edited by M. Baranger and E. Vogt Plenum, New York, p. 67.

[19] G. Rosensteel, D.J. Rowe (1977) Phys. Rev. Lett. 3810.

[20] M.A. Caprio, P.J. Fasano, P. Maris, A.E. McCoy, J.P. Vary (2020) Eur. Phys. J. A 56 120.

[21] A.E. McCoy, M.A. Caprio, T. Dytrych, P.J. Fasano (2020) Phys. Rev. Lett. 125 102505.

[22] R. Zbikowski, C.W. Johnson, A.E. McCoy, M.A. Caprio, P.J. Fasano (2021) J. Phys. $G 48075102$.

[23] M.A. Caprio, P.J. Fasano, A.E. McCoy, P. Maris, J.P. Vary (2019) Bulg. J. Phys. 46 445.

[24] A.M. Shirokov, I.J. Shin, Y. Kim, M. Sosonkina, P. Maris, J.P. Vary (2016) Phys. Lett. B 76187.

[25] M.A. Caprio, A.E. McCoy, P.J. Fasano (2020) J. Phys. G 47122001.

[26] J.P. Draayer, Y. Leschber, S.C. Park, R. Lopez (1989) Comput. Phys. Commun. 56 279.

[27] T. Dytrych, P. Maris, K.D. Launey, J.P. Draayer, J.P. Vary, D. Langr, E. Saule, M.A. Caprio, U. Catalyurek, M. Sosonkina (2016) Comput. Phys. Commun. 207202. 


\section{M.A. Caprio, A.E. McCoy, P.J. Fasano, T. Dytrych}

[28] O. Castaños, J.P. Draayer, Y. Leschber (1988) Z. Phys. A 32933.

[29] R.R. Whitehead (1980) In: "Theory and Applications of Moment Methods in ManyFermion Systems", edited by B.J. Dalton, S.M. Grimes, J.P. Vary, S.A. Williams (Plenum, New York), p. 235.

[30] C.W. Johnson (2015) Phys. Rev. C 91034313.

[31] V.G. Gueorguiev, J.P. Draayer, C.W. Johnson (2000) Phys. Rev. C 63014318.

[32] R.A. Herrera, C.W. Johnson (2017) Phys. Rev. C 95024303.

[33] F.Q. Luo, M.A. Caprio, T. Dytrych (2013) Nucl. Phys. A 897109.

[34] H.M. Aktulga, C. Yang, E.G. Ng, P. Maris, J.P. Vary (2013) Concurrency Computat.: Pract. Exper. 262631.

[35] M. Shao, H.M. Aktulga, C. Yang, E.G. Ng, P. Maris, J.P. Vary (2018) Comput. Phys. Commun. 2221. 